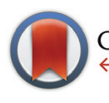

CrossMark

Cite this: Food Funct., 2015, 6, 3634

\section{A study of starch gelatinisation behaviour in hydrothermally-processed plant food tissues and implications for in vitro digestibility}

\author{
Cathrina H. Edwards, ${ }^{a}$ Frederick J. Warren, $\dagger^{\mathrm{a}}$ Grant M. Campbell, $t^{\mathrm{b}}$ Simon Gaisford, ${ }^{\mathrm{c}}$ \\ Paul G. Royall, ${ }^{d}$ Peter J. Butterworth ${ }^{a}$ and Peter R. Ellis*a
}

Received 22nd June 2015, Accepted 29th August 2015 DOI: $10.1039 / c 5 f 000754 b$ www.rsc.org/foodfunction

\begin{abstract}
The aim of this study was to investigate the role of the plant food matrix in influencing the extent of starch gelatinisation during hydrothermal processing, and its implications for starch digestibility. Differential scanning calorimetry (DSC) was used to provide a detailed examination of the gelatinisation behaviour of five distinct size fractions (diameters $<0.21$ to $2.58 \mathrm{~mm}$ ) of milled chickpea and durum wheat. Gelatinisation parameters were obtained from the DSC thermograms and concomitant microscopy analyses were performed. The estimated terminal extent of gelatinisation (TEG) was compared with our previously published data for in vitro starch digestibility of the same food materials. We observed clear differences in the gelatinisation behaviour of matched size-fractions of chickpeas and durum wheat. In chickpea materials, the TEG values (34-100\%) were inversely related to particle size, whereas in durum wheat, no sizedependent limitations on TEG were observed. The TEG values were completely consistent with the extent of starch amylolysis in all size fractions of both durum wheat and chickpea. Microstructural analysis following hydrothermal processing confirmed the presence of some partially gelatinised birefringent starch within intact chickpea cells. Birefringent starch granules were not present in any of the processed fractions of durum wheat. The differences in gelatinisation behaviour of these plant species seem to reflect the individual cell wall properties of these materials. These findings demonstrate the applicability of DSC to real food materials to provide insight into the mechanisms by which the food matrix (particularly the plant cell walls) influences gelatinisation, and consequently, starch amylolysis.
\end{abstract}

\section{Introduction}

Starch is the major source of carbohydrate in the diet and is present in a range of plant tissues. ${ }^{1}$ The gelatinisation of starch, caused by hydrothermal processing, is a crucially important functional property in the area of human nutrition, notably digestive physiology, as well as in a number of indus-

\footnotetext{
${ }^{a}$ Biopolymers Group, Diabetes and Nutritional Sciences Division, Faculty of Life Sciences and Medicine, King's College London, SE1 9NH London, UK. E-mail: p.ellis@kcl.ac.uk; Fax: +44 (0)20 7848 4171; Tel: +44 (0)20 78484238 ${ }^{b}$ Satake Centre for Grain Process Engineering, School of Chemical Engineering and Analytical Science, University of Manchester, M13 9PL Manchester, UK ${ }^{c}$ Pharmaceutics, School of Pharmacy, University College London, WC1N 1AX London, $U K$

${ }^{d}$ Drug Delivery Group, Institute of Pharmaceutical Science, Faculty of Life Science and Medicine, King's College London, SE1 9NH London, UK

$\dagger$ Current address: Centre for Nutrition and Food Sciences, ARC Centre of Excellence in Plant Cell Walls, The University of Queensland, Brisbane, Queensland 4072, Australia.

¥Current address: School of Applied Sciences, University of Huddersfield, Queensgate, HD1 3DH, Huddersfield, UK.
}

trial processes. ${ }^{2}$ Although studies of purified starch have provided much needed insight into the mechanisms and structural basis of gelatinisation, ${ }^{3-5}$ many industrial uses (including, inter alia, pelleting of biomass and food processing) involve gelatinisation of starch while it is still entrapped in a plant matrix. ${ }^{6,7}$ The plant matrix, however, may impose considerable restrictions on the gelatinisation of entrapped starch, and results in the formation of starch granules with a distorted 'buckled saddle' shape. ${ }^{8,9}$ Despite the implications this common phenomenon may have on starch functionality and digestibility, few research workers have attempted to fully characterise this effect, or have tried to address the underlying mechanisms. $^{8,10-13}$

Gelatinisation occurs when starch is heated in excess water. During this process, water de-stabilises hydrogen bonds in the amorphous regions of the granules, enabling further water ingress which is accompanied by granular swelling. This leads to swelling and disruption of starch crystallites, resulting in an endothermic transition, and the $\alpha$-glucan chains in starch becoming more disordered (i.e. amorphous). ${ }^{14}$ The gelatinisation transition is accompanied by a loss of birefringent 
properties as the starch becomes more amorphous. ${ }^{4}$ Once gelatinised, starch no longer retains its original granular structure, and a collapsed granular envelope, often termed a 'granule ghost', can be observed using light microscopy. However, when starch is subjected to hydrothermal processing while entrapped inside the cells of edible plant tissues or other food matrices, distorted granules with a characteristic 'buckled-saddle shape' often occur. ${ }^{8,12}$ It has been suggested that this distorted granular shape results from restrictions on heat, water or space required for starch granular swelling, and thereby results in limited gelatinisation. ${ }^{8,10,11,13}$

The limited extent of starch gelatinisation in plant tissue has implications for its physico-chemical properties and can affect its dietary and commercial utilisation. The more amorphous structure of gelatinised starch signifies a greater availability of $\alpha$-amylase binding sites, which makes the substrate more susceptible to enzyme hydrolysis. ${ }^{15,16}$ This is particularly important for human and animal nutrition, because the rate and extent of starch digestion is a key determinant of the glycaemic response to starch-rich foods, which in turn is highly relevant to human health and farm animal productivity. ${ }^{17,18}$ Considering the vast differences in digestion kinetics between native and gelatinised starches, ${ }^{11}$ partial gelatinisation would be expected to have major implications for digestibility and postprandial glycaemia. However, detailed studies of the digestibility of foods containing distorted starch granules, arising from limited gelatinisation, have yet to be performed.

Numerous workers have demonstrated that the extent of starch gelatinisation can be manipulated by controlling a variety of factors that include water availability, heating conditions, and by the inclusion of non-starch components during processing. ${ }^{3,19-21}$ These previous studies, however, were all performed on purified starches and are not necessarily representative of gelatinisation events that occur within more complex food materials. Achieving predictable control of gelatinisation in plant tissues and other food matrices is of great interest to a number of industrial processes. However, the multiplicity of effects (e.g., heat and water ingress, polymer interactions, structural changes) accompanying hydrothermal processing of these heterogeneous materials presents a formidable challenge.

While differential scanning calorimetry (DSC) is an excellent technique for studying starch gelatinisation, the small volume of typical sample pans $(c a .1-200 \mu \mathrm{L})$ limits its use both for large samples (i.e. 1-10 mm particle size scale), and samples containing significant quantities of water. In this study, we utilise a DSC instrument that accommodates a relatively large sample volume $(1 \mathrm{~mL})$ but which still provides high resolution. This technique is very well-suited to observing thermal transitions in foods, and enables samples to be heated in excess water under conditions that are relevant to many industrial processing methods.

The aim of this study was to use DSC to determine quantitatively the role of the plant matrix in influencing the extent of starch gelatinisation during hydrothermal processing, and its implications for starch digestibility and postprandial glycaemia. We reported recently that starch digestion kinetics of processed durum wheat and chickpea tissues, which have wellknown differences in cell wall properties and glycaemic potential, ${ }^{22,23}$ were strongly influenced by the degree of starch encapsulation by plant cell walls. ${ }^{24}$ It was hypothesised that the structural integrity of these materials could also play a central role in influencing the gelatinisation of starch. In the present study, we examined the gelatinisation behaviour and the concomitant microstructural changes of the same milled chickpea and durum wheat materials used previously. ${ }^{24}$ Our comparison of gelatinisation behaviour and digestibility of starch within these two edible plant species provided insight into the mechanisms by which the plant matrix (particularly the cell walls) influenced gelatinisation, and consequently, starch amylolysis.

\section{Materials and methods}

\subsection{Food materials}

Chickpeas (Russian $c v$.) were donated from Poortman Ltd, London, UK, and durum wheat grains (Svevo $c v$.) were provided by Millbo, S.p.A, Italy and were described in detail previously. ${ }^{24}$ Starch was extracted from these materials by a method that has been described elsewhere, ${ }^{25,26}$ except that purification was performed in ethanol, rather than in $\mathrm{NaOH}$ or water. ${ }^{24}$

The preparation of milled-macroparticles has been described in detail previously. ${ }^{24}$ In brief, de-hulled or debranned peas or grains were roller-milled (STR-100 test roller mill, Satake Corporation, Hiroshima, Japan) and then separated into distinct size fractions using a series of sieves. For the current study, five distinct fractions were selected and these were denoted $<0.21,0.55,1.02,1.55$ and $2.58 \mathrm{~mm}$ according to the median of the upper and lower sieve apertures. The size ranges of the test fractions were selected to represent particle sizes that occur during food processing and in vivo mastication. For statistical and graphical purposes, the particle size was expressed on the basis of an estimated value for volume $(V)$ per surface area (SA). These values were calculated based on the assumption that all particles were cuboid, with a side length equivalent to the median particle diameters, as estimated from upper and lower sieve apertures.

The total starch content of all milled size fractions and starches was determined using a modified version of the Megazyme Total Starch AOAC 996.11 Method (DMSO format), as described elsewhere. ${ }^{24}$ Moisture contents were determined by oven-drying at $105{ }^{\circ} \mathrm{C}$ to a constant weight. Proximate analyses of the milled durum wheat and chickpea were performed by Premier Analytical Services (High Wycombe, UK) according to accredited in-house methods. In brief, samples were analysed for crude protein $(\mathrm{N} \times 6.25$, determined by Dumas procedure $^{27}$ ), lipid (by Werner-Schmidt process ${ }^{28}$ ), dietary fibre (determined gravimetrically by AOAC method 991.43), and ash (according to BS 4603:1970). 


\subsection{Microscopy}

For examination by light microscopy, cooked samples of chickpea and durum wheat macroparticles were immersed in Karnovsky's fixative $(1.6 \%, \mathrm{v} / \mathrm{v}$, formaldehyde and $2 \%, \mathrm{v} / \mathrm{v}$, glutaraldehyde, $0.08 \mathrm{M}$ sodium cacodylate, $\mathrm{pH}$ 7.2), and left to fix at room temperature for at least $24 \mathrm{~h}$. The samples were subsequently rinsed in sodium cacodylate buffer $(0.1 \mathrm{M})$, dehydrated through increasing concentrations of ethanol, and then infiltrated with freshly prepared firm Spurr resin mixture (embedding kit purchased from Sigma-Aldrich Co Ltd, Poole, Dorset, UK), using propylene oxide (99\%, v/v) as the transition solvent. Finally, resin-embedded samples were polymerized at $70 \pm 2{ }^{\circ} \mathrm{C}$ for $12 \mathrm{~h}$. The cured samples were trimmed and sectioned $(0.5-1.0 \mu \mathrm{m})$ on an Ultracut E, Reichert-Jung microtome mounted with a glass knife. Sections were stained with toluidine blue $(1 \%$, w/v, with $1 \%, w / v$, sodium borate) and viewed on a Zeiss Axioskop 2 mot plus light microscope (Carl-Zeiss, Cambridge, UK). Images were captured with a Zeiss AxioCam HRc and AxioVision v3.1 microscope software.

For scanning electron microscopy (SM), dry, uncooked samples were mounted on double-sided carbon tape on an aluminium stub and coated with gold in a Polaron E5100 sputter coating unit. Samples were viewed on a Hitachi S-3500N scanning electron microscope (FEI Company, Cambridge, UK) using a $20 \mathrm{KV}$ accelerating voltage.

Birefringence was assessed both before and directly after DSC by viewing samples on a Leitz Dialux ED22 microscope (Leica Microsystems Ltd, Milton Keynes, UK) fitted with crossed polarisers and a red $1(\lambda)$ compensator plate. For viewing, the samples were suspended in a drop of deionised water on a glass slide and sealed with a cover slip. Image acquisition was performed using a Qi Imaging QiFastCam camera and Q-capture pro software.

\subsection{Differential scanning calorimetry}

DSC analysis was performed using a Multi-Cell DSC (TA Instruments, Elstree, UK). Starch or milled material was weighed into $1.0 \mathrm{~mL}$ capacity Hastelloy® ampoules, to which was added $1.00 \mathrm{~g}$ of de-gassed, deionised water. The weight of milled material added was adjusted (on the basis of measured starch content) so that all pans contained approximately $50 \mathrm{mg}$ of starch. A pan containing only water (i.e. which contributes a significant thermal mass in the sample pans) was also included as a reference. Pans were hermetically sealed and gently shaken before loading into the DSC instrument, and the position of each sample within the chamber was alternated between replicate runs. Prior to heating, the instrument was equilibrated for $2.5 \mathrm{~h}$ at $22^{\circ} \mathrm{C}$, during which time the materials were soaked. The pans were then heated from $20{ }^{\circ} \mathrm{C}$ to $90{ }^{\circ} \mathrm{C}$ at $1{ }^{\circ} \mathrm{C} \mathrm{min}^{-1}$, held at $90{ }^{\circ} \mathrm{C}$ for a further $10 \mathrm{~min}$, then cooled back to $20{ }^{\circ} \mathrm{C}$ in a chamber constantly purged with nitrogen at a flow rate of $50 \mathrm{~mL} \mathrm{~min}^{-1}$. Triplicate measurements were performed on all samples.

\subsection{Processing and analysis of DSC data}

Overlay images of typical endothermic curves were generated using TA Universal Analysis 2000 software (version 4.5A, 2007@ TA Instrument - Waters LLC). Peak integration and estimation of gelatinisation parameters were performed using NanoAnalyze Data Analysis software (version 2.2.0, TA Instruments 2005(). Onset, peak, and conclusion temperatures (denoted $T_{\mathrm{o}}, T_{\mathrm{p}}, T_{\mathrm{c}}$ ) and the enthalpy of gelatinisation $\Delta_{\text {gel }} H$ $\left(\mathrm{J} \mathrm{g}^{-1}\right)$ were obtained from each thermogram as described elsewhere. $^{3}$ The terminal extent of gelatinisation (TEG) represents the proportion of starch within a sample that undergoes gelatinisation and was calculated from observed gelatinisation enthalpies as described in eqn (1). This equation was based on that of previous workers, ${ }^{21}$ except that we did not observe an enthalpy change on the second heating cycle, and therefore modified the equation to exclude the correction for residual enthalpy. ${ }^{21}$ This estimation of TEG requires the starch content of the sample to be known, and is based on the assumption that any energy absorbed by the sample upon heating is associated only with gelatinisation of starch. The exact weight of tissue and its starch content was accounted for in all calculations.

$$
\operatorname{TEG}(\%)=\frac{\Delta_{\text {gel }} H \text { of milled material }}{\Delta_{\text {gel }} H_{\text {sp }} \text { of purified starch }} \times 100
$$

where TEG was obtained from the enthalpy change $\left(\Delta_{\text {gel }} H\right.$, expressed as $\mathrm{J} \mathrm{g}^{-1}$ starch) associated with gelatinisation of starch entrapped within milled material divided by the specific enthalpy associated with gelatinisation of $1 \mathrm{~g}$ of purified starch $\left(\Delta_{\text {gel }} H_{\text {sp }} \mathrm{J} \mathrm{g}^{-1}\right.$, which represents $100 \%$ TEG) in excess water conditions.

\subsection{In vitro digestibility}

In vitro digestibility of the cooked chickpea and durum wheat materials used for DSC analysis was determined in our laboratory using a well-established starch digestibility assay method. ${ }^{24}$ In brief, hydrothermally processed materials were incubated with $8 \mathrm{nM}$ porcine-pancreatic $\alpha$-amylase in PBS $\left(37^{\circ} \mathrm{C}, \mathrm{pH} 7\right)$ with continuous mixing and aliquots collected from the digestion mixture were analysed for total reducing sugars (i.e. starch digestion products) using the Prussian blue assay. ${ }^{15}$ The digestibility curves obtained for the chickpea and durum wheat materials used in the present study have been published previously. ${ }^{24}$ In the present study, we express the total extent of starch digestion (denoted $C_{\infty}$ ), which was determined using logarithm of slope analysis, ${ }^{24,29}$ as a function of particle size and relate this to key gelatinisation parameters. The $C_{\infty}$ values were expressed as a percentage of total hydrolysable starch, in which the gelatinised purified chickpea and durum wheat starches were taken to represent maximum hydrolysis (i.e. 100\% hydrolysable starch).

\subsection{Statistical analysis}

Statistical tests were performed by SPSS Statistics (version 20 IBM $\odot$ Corp.) and graphs were produced in Sigma Plot (version 
12.0 Systat@ software Inc.). Pearson's correlation tests were used to study relationships between particle size, TEG and $C_{\infty}$. All values shown are means $(n \geq 3) \pm$ SEM unless otherwise specified.

\section{Results}

\subsection{Material characteristics}

Purified starches contained $97 \%(\mathrm{w} / \mathrm{w})$ starch (on a dry weight basis). The total starch content (means $\pm \mathrm{SD}$ ) of milled chickpea and durum wheat was $45 \pm 1.1$ and $71 \pm 3.1$, respectively, expressed on a g per $100 \mathrm{~g}$ dry weight basis. Proximate analysis indicated that milled durum wheat (mean of triplicate values \pm SEM, expressed per $100 \mathrm{~g}$ fresh weight) contained $10.7 \pm 0.0 \mathrm{~g}$ protein, $70.2 \pm 0.2 \mathrm{~g}$ available carbohydrate (starch and sugars), $1.7 \pm 0.0 \mathrm{~g}$ fat, $6.5 \pm 0.2 \mathrm{~g}$ dietary fibre, $0.9 \pm 0.1 \mathrm{~g}$ ash and $9.9 \pm 0.0 \mathrm{~g}$ moisture. Milled chickpeas contained $23.0 \pm$ $0.0 \mathrm{~g}$ protein, $37.5 \pm 0.6 \mathrm{~g}$ available carbohydrate (starch and sugars), $5.3 \pm 0.0 \mathrm{~g}$ fat, $22.6 \pm 0.7 \mathrm{~g}$ dietary fibre, $2.8 \pm 0.0 \mathrm{~g}$ ash and $8.7 \pm 0.0 \mathrm{~g}$ moisture.

\subsection{Microscopy}

SM confirmed that the vast majority of milled particles obtained from chickpea and durum wheat resembled a cuboid shape (Fig. 1A and B), with starch from fractured cells exposed on the particle surface (Fig. 1C and D). Also, light microscopy (Fig. 1E and F) confirmed that starch granules with a distorted shape were present within the intracellular matrix of hydrothermally processed chickpea and durum wheat. From these light micrographs it is also evident that the plant cell walls of chickpeas are considerably thicker than those of durum wheat, which may have implications for heat transfer and water ingress during hydrothermal processing.

\subsection{DSC}

Representative endotherms are shown for each size fraction in Fig. 2. Peaks of chickpea materials were generally narrower than those of durum wheat. Gelatinisation parameters are shown in Table 1. Chickpea starch gelatinisation occurred at a higher temperature $\left(T_{\mathrm{p}}=71 .{ }^{\circ} \mathrm{C}\right)$ than durum wheat starch $\left(T_{\mathrm{p}}=57 .{ }^{\circ} \mathrm{C}\right)$, but the $\Delta_{\text {gel }} H_{\mathrm{sp}}$ of the two purified starches (9.6 and $9.5 \mathrm{~J} \mathrm{~g}^{-1}$ for chickpea and durum wheat starch, respectively) were not significantly different.

In milled materials, gelatinisation occurred at a higher temperature, producing a $T_{\mathrm{p}} \sim 2-3{ }^{\circ} \mathrm{C}$ higher than that of the purified starches. In the same milled materials (i.e. excluding the purified starch), the $\Delta_{\text {gel }} H$ and TEG values of chickpea materials were significantly lower than those of durum wheat. In chickpea, $\Delta_{\text {gel }} H$ and TEG were strongly influenced by particle size $\left(P<0.001, r^{2}=0.91\right)$, whereas none of the gelatinisation parameters obtained for durum wheat materials correlated with size $(P>0.1)$. As a result of the higher gelatinisation temperature of chickpea starch, values obtained for $T_{\mathrm{o}}$, $T_{\mathrm{p}}$, and $T_{\mathrm{c}}$ were also significantly higher $(P<0.001)$ for chickpea than durum wheat materials. The presence of birefrin-
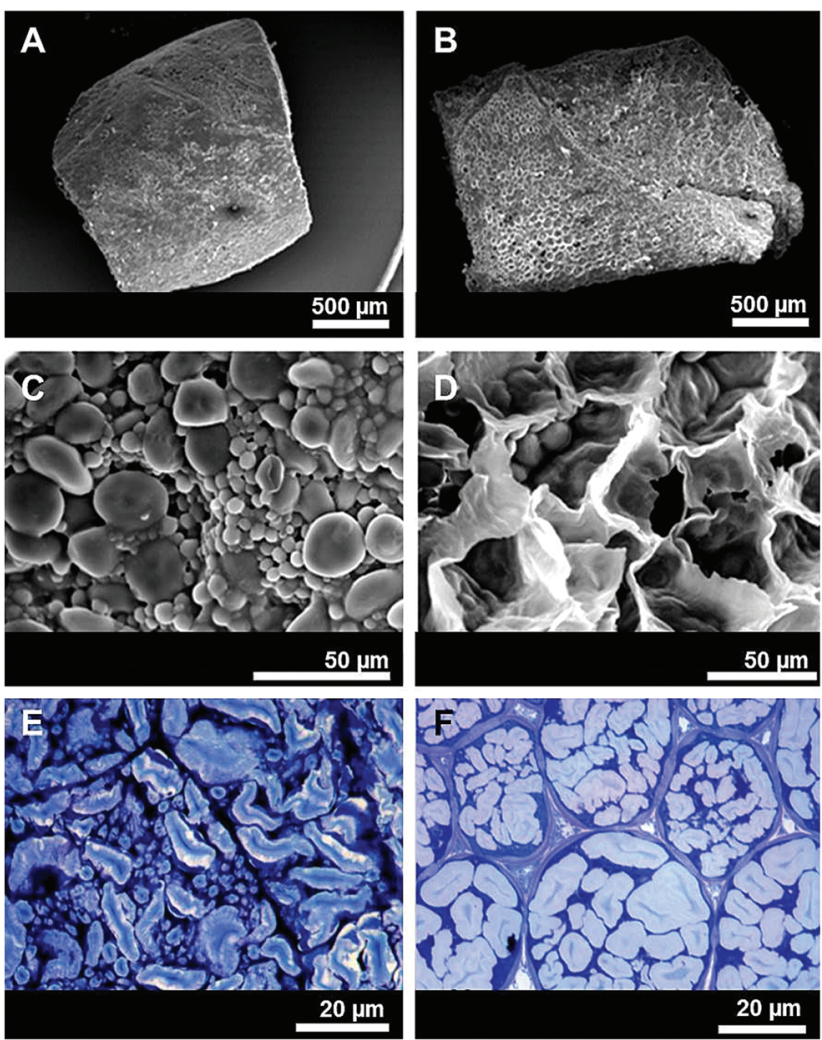

Fig. 1 Representative micrographs of durum wheat (left) and chickpea (right). Scanning electron micrographs (A-D) show the gross shape and surface of uncooked, milled macroparticles. Light micrographs ( $E$ and $F$ ) show the presence of starch granules with a distorted shape within intact plant cells, and are cross-sections from hydrothermally processed macroparticles.
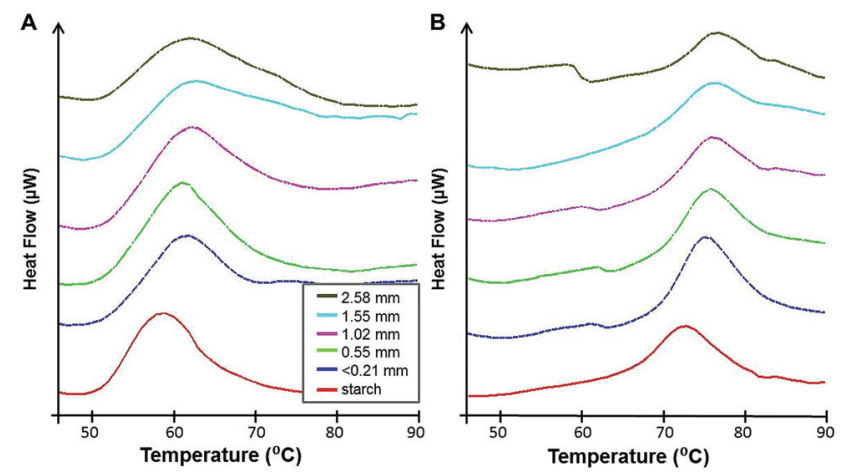

Fig. 2 Representative gelatinisation endotherms from different particle size fractions of durum wheat (A) and chickpea (B). The legend indicates median particle size and applies to both panels.

gence (shown in Fig. 3) in the chickpea samples only is consistent with these DSC results.

A plot of TEG against particle size (Fig. 4A) for both materials highlights the differences in gelatinisation behaviour between the two plant species. In milled chickpea samples, 
Table 1 Gelatinisation parameters of milled size fractions of chickpea and durum wheat ${ }^{a}$

\begin{tabular}{|c|c|c|c|c|c|c|c|}
\hline Sample & & $V / \mathrm{SA}(\mathrm{mm})$ & $T_{\mathrm{o}}\left({ }^{\circ} \mathrm{C}\right)$ & $T_{\mathrm{p}}\left({ }^{\circ} \mathrm{C}\right)$ & $T_{\mathrm{c}}\left({ }^{\circ} \mathrm{C}\right)$ & $\Delta_{\text {gel }} H\left(\mathrm{~J} \mathrm{~g}^{-1}\right.$ starch $)$ & TEG (\%) \\
\hline \multicolumn{8}{|c|}{ Chickpea } \\
\hline Starch & $(n=3)$ & 0.0 & $62.7 \pm 0.3$ & $71.7 \pm 0.4$ & $82.4 \pm 0.4$ & $9.6 \pm 0.0$ & $100.0 \pm 0.4$ \\
\hline 0.55 & $(n=3)$ & 0.092 & $66.7 \pm 0.3$ & $75.0 \pm 0.0$ & $83.0 \pm 0.0$ & $6.1 \pm 0.4$ & $63.5 \pm 3.8$ \\
\hline 1.02 & $(n=3)$ & 0.169 & $67.0 \pm 0.6$ & $75.0 \pm 0.0$ & $83.0 \pm 0.0$ & $5.1 \pm 0.3$ & $52.6 \pm 3.0$ \\
\hline 1.55 & $(n=4)$ & 0.258 & $68.3 \pm 0.0$ & $75.0 \pm 0.5$ & $83.0 \pm 0.0$ & $3.9 \pm 0.2$ & $40.0 \pm 3.8$ \\
\hline 2.58 & $(n=3)$ & 0.429 & $68.3 \pm 0.9$ & $75.3 \pm 0.3$ & $82.3 \pm 0.3$ & $3.3 \pm 0.3$ & $34.4 \pm 2.6$ \\
\hline$<0.21$ & $(n=3)$ & 0.018 & $51.4 \pm 0.3$ & $60.0 \pm 0.0$ & $72.0 \pm 0.6$ & $10.0 \pm 0.3$ & $105.5 \pm 3.6$ \\
\hline 0.55 & $(n=3)$ & 0.092 & $49.1 \pm 0.0$ & $60.4 \pm 0.3$ & $73.1 \pm 1.2$ & $9.9 \pm 0.4$ & $103.5 \pm 4.3$ \\
\hline 1.02 & $(n=3)$ & 0.169 & $50.8 \pm 0.3$ & $60.4 \pm 0.3$ & $72.8 \pm 0.7$ & $9.6 \pm 0.2$ & $101.1 \pm 2.5$ \\
\hline 1.55 & $(n=3)$ & 0.258 & $50.4 \pm 0.7$ & $59.4 \pm 0.7$ & $71.7 \pm 0.3$ & $9.7 \pm 0.1$ & $102.3 \pm 1.3$ \\
\hline 2.58 & $(n=3)$ & 0.429 & $50.7 \pm 0.9$ & $61.0 \pm 0.6$ & $75.4 \pm 1.3$ & $8.1 \pm 0.5$ & $85.3 \pm 5.7$ \\
\hline
\end{tabular}

${ }^{a}$ Values are mean of triplicate runs \pm SEM, unless otherwise specified. Onset $\left(T_{\mathrm{o}}\right)$, peak $\left(T_{\mathrm{p}}\right)$ and concluding $\left(T_{\mathrm{c}}\right)$ temperatures of gelatinisation are shown. $\Delta_{\text {gel }} H$ is the enthalpy change associated with the gelatinisation of $1 \mathrm{~g}$ of starch. TEG is the terminal extent of gelatinisation, expressed as a percentage of total starch present.
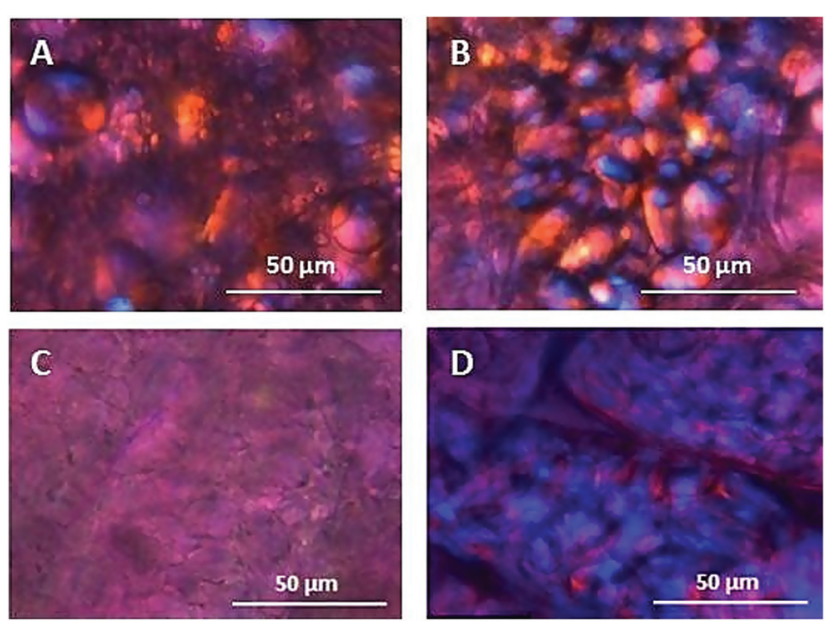

Fig. 3 Observations of birefringence in large particles of durum wheat (left) and chickpea (right) before (A and B) and after (C and D) DSC runs.

TEG decreased with increasing particle size. In durum wheat however, the observed enthalpy changes indicated that all of the starch (i.e., 100\%) underwent gelatinisation regardless of size, with the exception of the largest $(2.58 \mathrm{~mm})$ size fraction, where $85.3 \pm 5.7 \%$ of the starch was gelatinised. However, no birefringence could be observed in these largest particles of durum wheat after DSC, suggesting that, despite the DSC data, all the starch had in fact gelatinised (Fig. 3C). In comparison, birefringence was clearly evident in the same particle size $(2.58 \mathrm{~mm})$ of chickpea material after DSC (Fig. 3D).

\subsection{In vitro starch digestibility}

Digestibility data for purified starches and milled materials revealed a clear particle size effect (Fig. 4B). The highest digestibility was observed for purified starches and flour, with
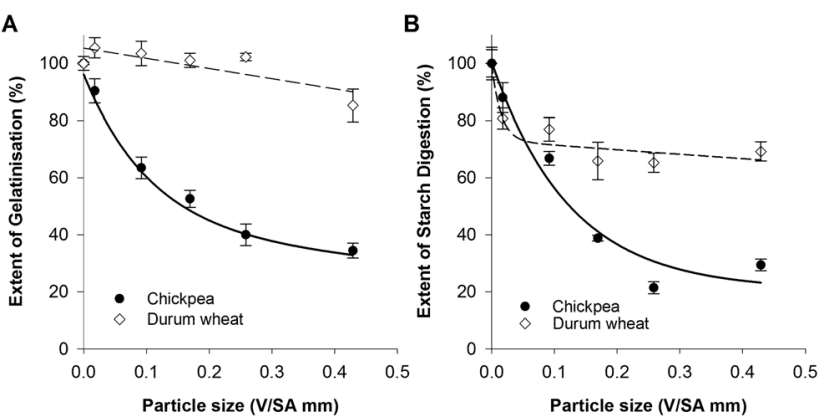

Fig. 4 Effect of particle size on the extent of gelatinisation (A) and digestibility (B) of starch in milled chickpea and durum wheat. Extent of gelatinisation (A) is represented by mean TEG values obtained by DSC, with error bars as SEM. Extent of starch digestion (B) is represented by normalised $C_{\infty}$ values for matched particle size fractions, with error bars as standard error of the estimate (SEE). Particle size is expressed as volume $\left(\mathrm{mm}^{3}\right)$ /surface area $\left(\mathrm{mm}^{2}\right)$. Curve-fits are provided just to illustrate the general relationships between particle size and gelatinisation or digestibility.

similar values for both botanical sources. The extent of digestion decreased with increasing particle size, with larger reductions in digestibility observed for chickpea materials than durum wheat.

The relationship between TEG and $C_{\infty}$ for chickpea and durum wheat samples of different particle sizes is shown in Fig. 5. A strong correlation was found between TEG and $C_{\infty}$ $\left(R^{2}=0.96\right.$, slope $=0.95 \%$ starch gelatinised per $\%$ starch digested) in chickpea size-fractions, whereas in durum wheat fractions, the trend between TEG and $C_{\infty}$ was less defined $\left(R^{2}=0.05\right.$, slope $=0.15 \%$ starch gelatinised per $\%$ starch digested $)$. The values for TEG $(>85 \%)$ and $C_{\infty}(>57 \%)$ of all size fractions of durum wheat were also mostly higher than matched size fractions of chickpea, particularly at larger particle sizes. 


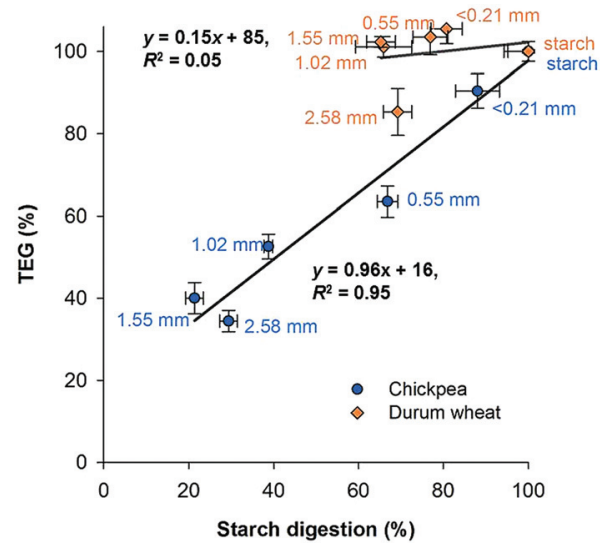

Fig. 5 Relationship between extent of gelatinisation and starch digestion in different particle size fractions of chickpea and durum wheat. Data points are mean $C_{\infty}$ and TEG values with horizontal error bars as $\mathrm{SEE}$, and vertical error bars as SEM, respectively. Data labels describe the nature of the material for which each data pair were obtained and shows particle diameter $(\mathrm{mm})$ or a material description. Fits were obtained by linear regression through an iterative process.

\section{Discussion}

We have used DSC to measure starch gelatinisation in purified starches and different size fractions of chickpea and durum wheat, and compared this with the extent of starch hydrolysis observed during in vitro digestion. A key finding of this study was that marked differences in the extent of gelatinisation and amylolysis were observed between chickpeas and durum wheat when the same size-manipulation was performed. In chickpea materials, the extent of gelatinisation was inversely related to particle size, resulting in potentially large and physiologically relevant differences in the extent of gelatinisation and starch digestion between milled fractions. The DSC thermograms for matched fractions of milled durum wheat, however, did not show a size-dependent effect, and nearly all the starch underwent gelatinisation in all size fractions. These findings provide new evidence that the effect of particle size on gelatinisation behaviour is not simply a result of available surface area per volume, but will also be related to the different physico-chemical properties of edible plant tissues, particularly with regard to the plant cell walls.

It is known that differences in starch characteristics influence gelatinisation behaviour, but the starches selected for this study were similar in many respects. Apart from the higher gelatinisation temperature of chickpea starch, chickpea and durum wheat starches had similar enthalpies of gelatinisation $\left(\Delta_{\text {gel }} H_{\mathrm{sp}}\right)$ and were both highly digestible (as indicated by their high $C_{\infty}$ values) after hydrothermal processing. Therefore, the differences in gelatinisation enthalpies between matched size fractions of milled chickpea and durum wheat are unlikely to be explained solely by inherent differences in starch properties.

One clear difference between the purified starches and all milled materials was the delayed onset of starch gelatinisation
$\left(T_{\mathrm{o}}\right)$ in the milled samples of both durum wheat and chickpea. This important finding suggests that there are structures and/ or components present in the milled fractions that hinder swelling and gelatinisation of starch granules, but are absent from and/or have no effect on the gelatinisation of purified starch. Apart from starch, the main components present in milled chickpea and durum wheat were found to be protein (23.0 and $10.7 \%$, respectively), dietary fibre (22.6 and 6.5\%, respectively), a reflection of the cell wall contents, and lipid (5.3 and $1.7 \%$, respectively). The vast majority of these components would have been removed as part of the extraction process to obtain the purified starch. However, the mere presence of these non-starch components does not provide a satisfactory explanation for the size-dependant changes observed.

We argue that the structure and properties of the food matrix are key factors that influence the conditions needed to gelatinise starch within plant foods. Previous evidence of the relationship between food structure and gelatinisation is described in the literature for a limited number of DSC studies of rice and pulses. ${ }^{10-13}$ These studies have demonstrated an increase in the extent of starch gelatinisation with increasing disruption of physical structure, which is probably explained by the greater exposure of released starch to water and heat during processing. ${ }^{10-13}$ Our approach using two different plant tissues provides further evidence of this complex relationship and of the implications for starch digestibility in different edible-plant materials. Any differences between matched sizefractions of durum wheat and chickpea are likely to reflect the different physico-chemical properties of the assembled plant tissue and their capacity to impose restrictions on starch gelatinisation. Thus, it seems there is some property of the chickpea tissue, not exhibited by durum wheat, which limits conditions for starch gelatinisation and therefore digestibility.

Partially swollen granules with a distorted shape have been observed within various food matrices (e.g., pasta, bread) or plant cells, and are thought to result from limitations imposed by the food matrix on the heat, water or space required for granular swelling and gelatinisation. ${ }^{8,10,11,30}$ There is evidence from studies of purified starch that if the water availability and thermal energy requirements for gelatinisation are not met, this results in restricted swelling of the granules and, consequently, limited digestibility. ${ }^{20,31,32}$ The conditions provided in our experimental set-up, however, should have provided favourable conditions for starch gelatinisation. The starch-rich materials were soaked in an excess of water over a $2.5 \mathrm{~h}$ period, which is a relatively long time considering the small size of the particles examined. We used modern DSC instrumentation and a very slow heating-rate, so that any limitations on heat transfer should have been largely overcome, and the gelatinisation process may be considered to have occurred under "quasiequilibrium" conditions, without kinetic limitations. ${ }^{33}$ Still, it is feasible that even with these provisions, the conditions for gelatinisation of starch granules entrapped within the food matrix may not have been met. Considering the heterogeneity of the plant materials used, it is possible that insufficient or uneven distribution of water and/or variations in heat transfer 
across individual particles hindered starch gelatinisation in a size-dependent manner. Restricted heat transfer or water ingress provides a reasonable explanation for limited gelatinisation in large particles, but is less convincing when it comes to explaining observations of distorted granules within isolated cells, ${ }^{8,12,13}$ where there is only a single cell wall barrier.

Another possibility is that the plant cell walls, or indeed intra-cellular components (e.g., protein), impose spatial restrictions on starch granule swelling. ${ }^{8,12}$ In some potatoes, for instance, the swelling of starch granules during gelatinisation exerts so much pressure on the surrounding cell walls that it can cause the cells to rupture. ${ }^{34}$ The cells of chickpeas and indeed other plant tissues are known to remain largely intact during processing..$^{8,13,35-37}$ Thus, it seems feasible that, within the confines of the intracellular matrix or indeed other complex food matrices (e.g. pasta), the pressure exerted by swelling of adjacent starch granules leads to deformations in granular shape. This mechanism would provide a satisfactory explanation for previous observations of distorted granules within a broad range of hydrothermally processed foods. ${ }^{8,9,12,38}$ The greater restrictions on starch gelatinisation within chickpeas (and probably other pulses) compared with durum wheat endosperm may be explained by the greater thickness and resilience of leguminous cell walls, which could impose greater restrictions on water ingress, heat transfer and space for granule swelling. The restrictive effects of plant cell walls also provides an explanation for the size-dependent effects on starch gelatinisation parameters, because the degree of starch encapsulation by cell walls varies in proportion to particle size. ${ }^{24,39}$ Overall, we take the view that all of the above mechanisms may be operative to greater or lesser extents, but further studies are needed to elucidate their individual importance.

The application of DSC techniques to studies of starch gelatinisation behaviour in real food materials should provide new insight into the effect of hydrothermal processing on starch properties and is therefore of relevance to human nutrition. In particular, the strong correlation between the extent of starch gelatinisation (TEG) and amylolysis $\left(C_{\infty}\right)$ implies that DSC may be used to predict starch digestibility. This is unsurprising given that gelatinisation is known to markedly increase the susceptibility of purified starch to amylolysis; ${ }^{15}$ however, the mechanistic basis for this relationship in a heterogeneous food matrix is more complex.

\section{Conclusions}

The plant tissue matrix clearly influences the degree and time course of starch gelatinisation, with likely implications for starch digestibility and postprandial glycaemia. On the whole, these results clearly highlight the importance of the impact of the food matrix on the swelling and gelatinisation processes. In particular, they point to an urgent need for further understanding of the effects of water availability and heat transfer on gelatinisation behaviour in a much broader range of starch- containing plant tissues. Moreover, we need a better understanding of the role played by different cell wall structures and individual intracellular non-starch components (e.g. proteins) in influencing starch gelatinisation. Such studies as presented here contribute to the developing area of study, and the approach outlined needs to be applied to controlled but varied starch systems and foods. Considering the important nutritional role of starch-rich foods, we envisage that this work is highly relevant to the development of a range of novel ingredients and functional foods, with potential applications in obesity management, colonic health, and the management and prevention of cardiometabolic diseases. ${ }^{40}$

\section{Conflict of interest}

No conflicts of interest are declared for any of the authors.

\section{Acknowledgements}

We thank Mary-Jo Searle (King's College London, UK) for technical assistance with starch determinations, and the Centre for Ultrastructural Imaging (King's College London, UK) for assistance with microstructural analysis. Thanks are also extended to Bruno Boggini (Millbo S.p.A., Trecate, Italy) and Jeremy Isaacs (Poortman Ltd, London, UK) for providing the durum wheat and chickpeas and also to Mike Jordan (Premier Analytical Services Ltd, UK) for providing proximate analysis data for these materials. The project was funded by the BBSRC, UK (DRINC BB/H004866/1) and C. H. E. was in receipt of a BBSRC CASE studentship award with Premier Foods (UK) as an industrial partner.

\section{References}

1 T. L. Wang, T. Y. Bogracheva and C. L. Hedley, J. Exp. Bot., 1998, 49, 481-502.

2 S. Jobling, Curr. Opin. Plant Biol., 2004, 7, 210-218.

3 T. Y. Bogracheva, Y. L. Wang, T. L. Wang and C. L. Hedley, Biopolymers, 2002, 64, 268-281.

4 J. W. Donovan, Biopolymers, 1979, 18, 263-275.

5 S. Wang and L. Copeland, Food Funct., 2013, 4, 1564-1580.

6 N. Kaliyan and R. Vance Morey, Biomass Bioenergy, 2009, 33, 337-359.

7 H. Röper, Starch.Stärke, 2002, 54, 89-99.

8 P. Würsch, S. Del Vedovo and B. Koellreutter, Am. J. Clin. Nutr., 1986, 43, 25-29.

9 A. Fardet, C. Hoebler, P. M. Baldwin, B. Bouchet, D. J. Gallant and J. L. Barry, J. Cereal Sci., 1998, 27, 133145.

10 E. T. Champagne, W. E. Marshall and W. R. Goynes, Cereal Chem., 1990, 67, 570-574.

11 W. E. Marshall, Cereal Chem., 1992, 69, 632-636.

12 T. Fujimura and M. Kugimiya, Starch/Stärke, 1994, 46, 374378. 
13 Y. Brummer, M. Kaviani and S. M. Tosh, Food Res. Int., 2015, 67, 117-125.

14 D. Cooke and M. J. Gidley, Carbohydr. Res., 1992, 227, 103112.

15 S. L. Slaughter, P. R. Ellis and P. J. Butterworth, Biochim. Biophys. Acta, Gen. Subj., 2001, 1525, 29-36.

16 A. J. Baldwin, D. L. Egan, F. J. Warren, P. D. Barker, C. M. Dobson, P. J. Butterworth and P. R. Ellis, Biomacromolecules, 2015, 16, 1614-1621.

17 J. Holm, I. Lundquist, I. Björck, A. C. Eliasson and N. G. Asp, Am. J. Clin. Nutr., 1988, 47, 1010-1016.

18 J. Parada and J. M. Aguilera, J. Food Sci., 2009, 74, E34E38.

19 P. A. Perry and A. M. Donald, Carbohydr. Polym., 2002, 49, 155-165.

20 N. Roder, C. Gerard, A. Verel, T. Y. Bogracheva, C. L. Hedley, P. R. Ellis and P. J. Butterworth, Food Chem., 2009, 113, 471-478.

21 M. Fukuoka, K.-i. Ohta and H. Watanabe, J. Food Eng., 2002, 53, 39-42.

22 K. Foster-Powell, S. H. Holt and J. C. Brand-Miller, Am. J. Clin. Nutr., 2002, 76, 5-56.

23 C. Brett and K. Waldron, Physiology and biochemistry of plant cell walls, Chapman \& Hall, London, 1996.

24 C. H. Edwards, F. J. Warren, P. J. Milligan, P. J. Butterworth and P. R. Ellis, Food Funct., 2014, 5, 2751-2758.

25 J. Vansteelandt and J. A. Delcour, Starch/Stärke, 1999, 51, 73-80.

26 D. Güzel and S. Sayar, Food Res. Int., 2010, 43, 21322137.
27 Manual of Methods for Wheat and Flour Testing, Guideline No. 3, ed. C. Anderson and S. Salmon, Camden and Chorleywood Food Research Association, 1999.

28 R. S. Kirk and R. Sawyer, Pearson's Composition and Analysis of Foods, Addison Wesley Longman Ltd., Essex, 1991.

29 P. J. Butterworth, F. J. Warren, T. Grassby, H. Patel and P. R. Ellis, Carbohydr. Polym., 2012, 87, 2189-2197.

30 K. Srikaeo, J. E. Furst, J. F. Ashton and R. W. Hosken, LWT-Food Sci. Technol., 2006, 39, 528-533.

31 P. A. Perry and A. M. Donald, Int. J. Biol. Macromol., 2000, 28, 31-39.

32 B. Svihus, A. K. Uhlen and O. M. Harstad, Animal Feed Sci. Technol., 2005, 122, 303-320.

33 N. I. Davydova, S. P. Leont'ev, Y. V. Genin, A. Y. Sasov and T. Y. Bogracheva, Carbohydr. Polym., 1995, 27, 109-115.

34 I. Shomer, Carbohydr. Polym., 1995, 26, 47-54.

35 T. Grassby, C. H. Edwards, M. Grundy and P. R. Ellis, in Stability of Complex Carbohydrate Structures: Biofuels, Foods, Vaccines and Shipwrecks, ed. S. E. Harding, Royal Society of Chemistry, 2013.

36 T. Berg, J. Singh, A. Hardacre and M. J. Boland, Carbohydr. Polym, 2012, 87, 1678-1688.

37 C. Melito and J. Tovar, Food Chem., 1995, 53, 305-307.

38 M. B. Dürrenberger, S. Handschin, B. Conde-Petit and F. Escher, Lebensm.-Wiss. Technol., 2001, 34, 11-17.

39 T. Grassby, D. R. Picout, G. Mandalari, R. M. Faulks, C. W. C. Kendall, G. T. Rich, M. S. J. Wickham, K. Lapsley and P. R. Ellis, Food Funct., 2014, 5, 3096-3106.

40 N.-G. Asp, J. M. M. van Amelsvoort and J. G. A. J. Hautvast, Nutr. Res. Rev., 1996, 9, 1-31. 